\title{
Microchip Isoelectric Focusing Applications
}

\author{
Markéta Vlčková§a and Maria A. Schwarz ${ }^{\star a, b}$ \\ §SCS Mettler-Toledo Award Winner (Oral Presentation)
}

\begin{abstract}
This review summarizes all available publications on isoelectric focusing carried out on planar microfabricated devices. Characteristic features of microchip isoelectric focusing (MC-IEF), such as resolution independence of separation length and compression of $\mathrm{pH}$ gradient, are discussed. An overview of materials used for microchip fabrication and developed detection strategies is given. Accomplishments in on-chip coupling of MC-IEF with other electrophoretic separation techniques or with mass spectrometry are briefly described. The review ends with alternative approaches to MC-IEF separation in terms of unusual sample introduction, kinds of support used for IEF separation, and in manner in which the $\mathrm{pH}$ gradient is generated.
\end{abstract}

Keywords: Electrophoresis · Isoelectric focusing · Microfluidics · Proteomics · Whole-column imaging detection

\section{Introduction}

Isoelectric focusing (IEF) is an electrophoretic separation technique with high resolution power used to separate amphoteric compounds, in particular peptides and proteins. ${ }^{[1]}$ The amphoteric compounds are separated on the basis of their isoelectric point ( $\mathrm{pI}$ ) in a $\mathrm{pH}$ gradient formed by a mixture of special amphoteric buffers, socalled ampholytes. Various wide-pH-range and narrow-pH-range ampholytes mixtures from different suppliers are commercially

\footnotetext{
${ }^{\star}$ Correspondence: Dr. M. A. Schwarz ${ }^{a, b}$

Tel.: + 41616866523

Fax: + 41612671005

E-mail: Maria.Schwarz@unibas.ch,

Maria.Schwarz@solvias.com

aDepartment of Chemistry

University of Base

Spitalstrasse 51

$\mathrm{CH}-4056$ Basel

bSolvias AG

WKL-127.2.40

Klybeckstrasse 191

Postfach

$\mathrm{CH}-4002$ Basel
}

available in order to optimize a given IEF separation.

The ampholytes build the $\mathrm{pH}$ gradient in the electric field, which is applied along a slab gel, a capillary or, more recently, along a separation channel on the microchip. Before the electric field is applied, the separation device is filled with a solution of uniform $\mathrm{pH}$ which contains a mixture of ampholytes and a sample. The electrode reservoirs are filled with an acid and a base, respectively. Upon application of the electric field, the negatively charged ampholytes move towards the anode, and the positively charged ones to the cathode. They gradually align themselves between the cathode and the anode according to their $\mathrm{pI}$ values, and form a stable $\mathrm{pH}$ gradient. Simultaneously, sample components (proteins, peptides) are separated and focused to those positions of the $\mathrm{pH}$ gradient which correspond to their $\mathrm{pI}$ values. Thus, an estimation of the pI of the unknown sample components is possible with the help of pI markers of known pI values (available commercially). The experimental set-up of IEF performed in the narrow channel format (capillary, microchip) is schematically depicted in Fig. 1a.

Capillary isoelectric focusing (cIEF) offers many advantages over gel-based IEF, such as ease of automation, quantitation, lower consumption of reagents and faster analysis speed. ${ }^{[2]}$ Microchip isoelectric focusing (MC-IEF) results from the miniaturization of the cIEF and so the amount of reagents is further drastically reduced. ${ }^{[3]}$
Moreover, MC-IEF may be on-chip coupled with other processes, which can substantially simplify and speed up the analysis of complex samples. ${ }^{[4]}$ However, most of the commercial instruments for cIEF, and also certain MC-IEF configurations, rely on single-point, on-column optical detection (UV/VIS absorption, fluorescence) and hence do not enable direct detection of the focused proteins (unlike gel-based IEF). For the detection of the focused zones, the focusing process must be followed by a mobilization step. During mobilization all protein bands focused inside the capillary or the micro-channel are moved past the detection point. However, the mobilization leads to distortion of the $\mathrm{pH}$ gradient resulting in poor reproducibility, longer analysis time and poorer resolution. ${ }^{5]}$ To overcome this obstacle, various whole-column imaging detectors for cIEF and MC-IEF have been developed. ${ }^{[2,6]}$ A charged-coupled camera of such a detector images the entire capillary or separation channel, often in real time, and hence the need for mobilization is eliminated. Another benefit of imaged IEF is the possible monitoring of the focusing process, which provides valuable additional information. ${ }^{[2]}$

Since the first demonstration of IEF on the microchip in $1999,{ }^{[3,7]}$ a lot of effort has been put into the development of MC-IEF for various applications. This review gives an overview of all applications of MC-IEF which have been hitherto published. The classification of the applications into the sections in this review is based on new ac- 


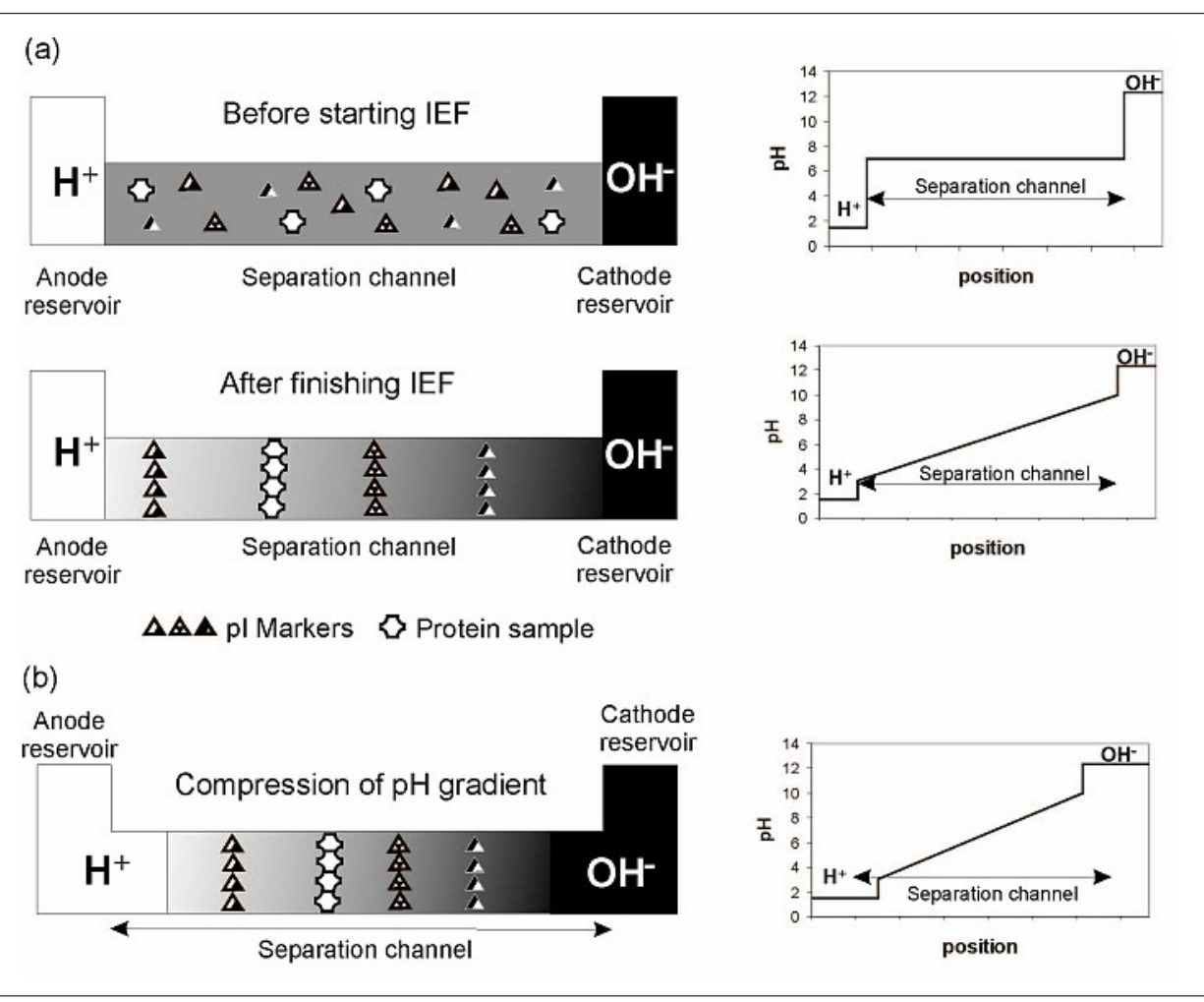

Fig. 1. Experimental set-up of IEF. Schematic drawing of (a) IEF process performed in a narrow channel format; (b) compression of $\mathrm{pH}$ gradient occurring during MC-IEF.

complishments, rather than on the type of analyzed protein. The first section covers first realizations of MC-IEF and contains three sub-sections referring to MC-IEF phenomena, detection systems for MC-IEF, and available chip materials, respectively. A section is devoted to applications dealing with on-chip integration of MC-IEF with other processes. Finally, the alternative approaches to MC-IEF are summarized in a separate section. The review ends with conclusions and a short outlook to the future.

\section{Realization of MC-IEF}

The early applications of MC-IEF were mostly aimed at the demonstration of feasibility of IEF separation on a microfluidic device. Hofmann et al. constructed a glass microchip for MC-IEF and compared three common mobilization methods. ${ }^{[7]}$ The electroosmotically driven mobilization occurring simultaneously with the focusing has been suggested for using with microchip due to its speed, compatibility with electroosmotic flow (EOF), and minimum instrumentation requirements. Concurrently, MC-IEF on the chip with UV absorption imaging detection has been demonstrated by Mao and Pawliszyn. ${ }^{[3]}$ The channels of the quartz chip applied for the IEF separation had to be coated with linear polyacrylamide to achieve good reproducibility and resolution. More recently, MC-IEF was performed on a plastic device; ${ }^{[8]}$ single-point LIF detection together with chemical mobilization was used for this MC-IEF separation. The same device has been also successfully applied to the study of protein-protein interactions causing a $\mathrm{pI}$ value change. ${ }^{[8]}$ Fast MC-IEF separation carried out on a plastic chip with ultra-short separation channel has been demonstrated by Han and Singh. ${ }^{[9]}$ Using a microchannel $4 \mathrm{~mm}$ long, IEF could be completed within $45 \mathrm{~s}$ without sacrificing the resolution. The resolution was not lowered while shortening the channel (at constant voltage), as follows from the resolution phenomenon occurring in IEF (see below).

\subsection{MC-IEF Phenomena}

During the first miniaturization efforts, it was confirmed experimentally as well as theoretically, that in IEF separations, the resolution does not depend on the separation length. ${ }^{[8-10]}$ Das and Fan studied this phenomenon in MC-IEF in detail.[10]

The minimum difference in $\mathrm{pI}$ values required for two proteins to be separated $\left(\Delta(\mathrm{pI})_{\min }\right)$ can be given by the following Eqn. first reported by Vesterberg and Svensson:[11]

$$
\Delta(\mathrm{pI})_{\min }=3 \sqrt{\frac{\mathrm{D} \cdot(\mathrm{d}(\mathrm{pH}) / \mathrm{dx})}{\mathrm{E} \cdot(-\mathrm{d} \mu / \mathrm{d}(\mathrm{pH}))}}
$$

where $\mathrm{D}$ is the diffusion constant of the protein, $\mathrm{d}(\mathrm{pH}) / \mathrm{dx}$ is the $\mathrm{pH}$ gradient, $\mathrm{E}$ is electric field strength, and $\mathrm{d} \mu / \mathrm{d}(\mathrm{pH})$ is the mobility slope at each pI value. The following assumptions have been used in the derivation of Eqn. (1). First, $\mathrm{pH}$ gradient is continuous and the applied electric field is constant. Second, both proteins have the same diffusion coefficient. Third, the two adjacent proteins are considered separated when the positions of their peak maximums differ by three standard deviations. Fourth, there are no effects resulting from EOF, hydrodynamic siphoning, and other such movements. Finally, there is no Joule heating due to high electric currents.

When a constant voltage, $\mathrm{V}$, is applied and a uniform $\mathrm{pH}$ gradient is used, then $\mathrm{E}$ $=\mathrm{V} / \mathrm{L}$ and $\mathrm{d}(\mathrm{pH}) / \mathrm{dx}=\Delta \mathrm{pH} / \mathrm{L}$, where $\mathrm{L}$ is separation length. Consequently, Eqn. (1) may be simplified to Eqn. (2):

$$
\Delta(\mathrm{pI})_{\min }=\sqrt[3]{\frac{\mathrm{D} \cdot \Delta \mathrm{pH}}{\mathrm{V} \cdot(-\mathrm{d} \mu / \mathrm{d}(\mathrm{pH}))}}
$$

in which there is no term related to the distance. By shortening the channel length, a higher electric field is applied when the voltage remains the same and the peaks become sharper. Consequently, the IEF separation resolution is not sacrificed (at the same voltage $\mathrm{V}$ ) with the decreasing channel length. Naturally, the application of a higher separation voltage leads to a higher resolution (lower $\Delta(\mathrm{pI})_{\min }$, see Eqn. (2)). Additionally, a linear relationship between the focusing time and the inverse of the electric field strength has been established. ${ }^{[10]}$

Regarding the resolution dependence on separation voltage, Stoyanov et al. ${ }^{[12]}$ have demonstrated that above a certain electric field (similar to $300 \mathrm{~V} / \mathrm{cm}$ ), the resolution does not increase with the increasing voltage anymore and even lowers. This observation has been ascribed to the detrimental effects of Joule heating at higher electric field, which has led to a band-broadening and breakdown of a gel used to minimize the EOF.

Another phenomenon relating to MCIEF is the compression of $\mathrm{pH}$ gradient, which was for the first time reported by Cui et al. ${ }^{[13]}$ With the help of imaged detection it was observed that the $\mathrm{pH}$ gradient in a microfluidic device is compressed to the middle of a channel rather then uniformly distributed in the whole channel (Fig. 1b). This compression effect is caused by electrolytes drawing into the microchannels and the degree of compression decreases with the channel length. ${ }^{[10]}$ High concentrations of methylcellulose or its derivative have been added to the aqueous sample solution $^{[6]}$ or to the reservoir solutions ${ }^{[13]}$ to increase the viscosity and thus to reduce the compression effect. 


\subsection{Detection for MC-IEF}

The following three detector types for MC-IEF may be distinguished: singlepoint on-column detectors, whole-column imaged detectors, and special off-column detectors, respectively. Obviously, most efforts have been devoted to the development of whole-column imaged detection, as the problematic mobilization of the focused zones could be circumvented with this detector type. Moreover, the planar arrangement and short separation length make the practical realization of the imaged detection less demanding compared to cIEF. Imaged UV absorption detectors ${ }^{[3,14]}$ as well as various imaged fluorescence detectors have been developed for MC-IEF. In addition to lamp ${ }^{[15]}$ and laser (laser-induced fluorescence detection), [16,17] also organic light emitting diodes ${ }^{[6]}$ have been used as the light source for fluorescence detection. The imaging is usually realized by focusing the light on the whole channel and sensing through charged-coupled devices (CCD). Alternatively, a fast scanning fluorescence detector, moving along the channel, has been applied for MC-IEF. ${ }^{[15,16]}$

The single-point on-column detectors measure mostly fluorescence. ${ }^{[7,8]}$ In addition to fluorescence, a selective and sensitive chemiluminiscence detector has been developed for single-point detection of heme proteins separated by MC-IEF.[18] In relation to the single-point detection, a novel pressure on-chip mobilization using a diaphragm pump has been reported,[19] which enables a precise control of the mobilization and facilitates the connection of the IEF to second dimension separation technique.

Special off-column detection represents the demanding connection of MC-IEF to a mass spectrometer (MS). MC-IEF was successfully on-chip coupled to electrospray ionization (ESI), followed by ion-trap MS, as demonstrated by Wen et al. ${ }^{[20]}$ Recently, MC-IEF has been coupled with matrix-assisted laser desorption/ionization (MALDI) time-of-flight MS, using a pseudo-closed channel as an interface. [21] The pseudoclosed channel, which served for MC-IEF separation, was formed between two plastic wafers, the base and the cover, respectively. After removal of the cover, a buffer solution was evaporated from the base, and then the matrix solution was added. In this way the laser reached the separated proteins directly on-chip without an elution step. In a similar approach demonstrated by Fujita et al., ${ }^{[22]}$ a chip covered with removable resin tape has been applied for MC-IEF. After finishing the MC-IEF separation, the chip was immediately frozen in order to fix separated proteins in their position. Then the tape was removed, matrix solution was added, and the detection by MALDI-MS was performed.
The connection of MC-IEF with MS might be considered as a special case of on-chip integration of more processes as it enables a high-throughput two dimensional (2D) mapping. In the first dimension, sample proteins are analyzed in terms of their $\mathrm{pI}$ by MC-IEF, and in the second dimension, the samples are measured in terms of their mass-to-charge ratio by MS.[22]

\subsection{Chip Materials}

Besides traditional glass chips, MCIEF separations have also been often performed in cheaper plastic chips and, alternatively also in combined glass/plastic chips (one layer glass, second layer plastic $\left.{ }^{[6,18,19]}\right)$. In the case of plastic chips, poly(dimethylsiloxane) (PDMS) is the most often used polymeric material, $[9,13,23]$ but the use of other polymeric materials such as poly(methyl metacrylate) (PMMA) ${ }^{[8,24]}$ or polycarbonate $(\mathrm{PC})^{[23,25]}$ has also been reported.

An interesting alternative material for chip fabrication for MC-IEF is cyclic olefin copolymer (COC) due to its ease of fabrication, low cost and solvent resistance. ${ }^{[26]}$ The unfavorable protein adsorption normally associated with this material was suppressed by covering the surface of COC device by polyacrylamide. ${ }^{[26]}$ Covering the inner chip walls by polyacrylamide increased the hydrophilicity of the walls, and at the same time suppressed also the EOF, as desired for MC-IEF measurements with imaged detection. Alternatively, the EOF on COC device was kept low by dynamic coating using a mixture of hydroxyethylcellulose and hydroxypropylcellulose. ${ }^{[10]}$ To further suppress protein adsorption onto this device, only the second run of MC-IEF separation was evaluated.

The glass chips for the MC-IEF are usually coated, either dynamically ${ }^{[18]}$ or permanently using polyacrylamide. ${ }^{[3,27]}$ An unusual approach to the permanent coating is the modification of the glass surface using plasma-polymerized films, as reported by Tsai et al. ${ }^{[28]}$ Acetonitrile as well as hexamethyldisiloxane (HMDS) monomers were used for plasma polymerization to modify the glass surface; the stability of the coating was substantially better in case of hydrophobic HMDS films. ${ }^{[28]}$

\section{On-chip Integration of MC-IEF with other Processes}

One of the attractive features of chip technology is the potential of integration of more processes into one device with the goal to eventually develop a so-called micro-total analysis system ( $\mu$-TAS), a miniaturized flow system for fast, fully automated analysis. Due to the relative newness of MC-IEF technology, MC-IEF has so far been coupled only to another electrophoretic separation technique or to the MS detector. As the connection of MC-IEF with MS has already been described in one of the previous sections (detection for MC-IEF), this section summarizes available reports on coupling of MC-IEF to another electrophoretic process.

The integration of MC-IEF with other electrophoretic technique was mostly aimed at development of a 2D separation device with two independent separation mechanisms, which are employed sequentially. Typically, the MC-IEF step serves as the first dimension followed by another electrophoretic separation step, which can either be free solution electrophoresis ${ }^{[29,30]}$ or gel electrophoresis. ${ }^{24,25,30]}$ The most challenging task is the transfer of proteins between the two dimensions, which is achieved through a special design of the chip in combination with a complicated voltage control ${ }^{[24,25,29]}$ or, alternatively, with a pressure control using a system of microvalves. ${ }^{[30]}$

A slightly different approach to the on-chip integration of more processes represents the coupling of several stages of MC-IEF separation in series. ${ }^{[31]}$ First, proteins are focused in a straight channel using broad-range ampholytes. Then, segments of the first channel are refocused in secondary channels branched from the first channel at T-junctions. A significantly higher resolution was achieved during refocusing of the tested proteins in the secondary channels due to shallower $\mathrm{pH}$ gradient and higher electrical field gradient in comparison to the MC-IEF separation in the first channel.

\section{Alternative Approaches to MC-IEF}

In addition to the standard set-up of MC-IEF (summarized in Fig. 1), a number of MC-IEF separations with non-standard arrangement of the IEF process have been developed. The unusual approaches to MCIEF differ from the classical arrangement:

i) in terms of sample introduction;

ii) in the kind of separation device used for IEF separation;

iii) in the manner of generation of $\mathrm{pH}$ gradient.

i) In a standard MC-IEF arrangement, the channel is filled with the mixture of ampholytes and sample before starting the focusing process. An alternative, dynamic sample introduction for MCIEF has been developed in order to increase sample loading and thereby also the concentration of the analytes in the focused zones. ${ }^{[23]}$ In this dynamic approach, the analytes (proteins or peptides) are initially present in electrode reservoirs and during the separation continuously migrate into the channel, encounter the $\mathrm{pH}$ gradient established 
by the ampholytes and undergo the focusing. Sample loading capacity can be directly controlled by the injection time and the applied electric field strength; however the discrimination between the analytes by electrokinetic injection cannot be avoided (the loading of analyte with higher electrophoretic mobility will be greater compared to the analyte with lower mobility).

ii) The standard MC-IEF separation takes place in a narrow separation microchannel. In contrast, a flat bed is used for separation in the method of free flow $M C$ $I E F$, introduced by $\mathrm{Xu}$ et al. ${ }^{[32]}$ In free flow IEF, a wide stream of a mixture of ampholytes and sample is continuously admitted at one end of the separation bed under laminar pressure flow conditions. An electric field is applied perpendicular to the pressure driven flow with the help of side channels. These side channels connect the separation bed with electrode compartments filled with an acid and a base, respectively, and serve for applying an electric field (Fig. 2a). Sample components migrate through the separation bed transversally until they reach their isoelectric points and thereafter form narrow parallel lines. Consequently, free flow MC-IEF is applied mainly to increase the concentration of the analytes. However, the side channels cause a significant potential loss and a loss of part of the $\mathrm{pH}$ gradient due to the spreading of ampholytes to them. To overcome this drawback, an improved device for free-flow MCIEF has been developed by Kohlheyer at el. [33] Two photopolymerizable membranes fabricated directly in the chip isolate the separation bed from the electrodes, yet allow the electrical contact due to their ion permeability. The design of the device has been further improved by employing multiple sheath flows and preseparated ampholytes, leading to a higher resolution and peak capacity of the free flow MC-IEF separation. ${ }^{[34]}$ Another non-standard separation device is utilized in so-called parallel IEF developed by Zilberstein et al. ${ }^{[35-37]}$ In the specially designed parallel device, the $\mathrm{pH}$ gradient is not created along the electric field, but across a membrane with a large amount of microscopic channels filled by gel droplets with different $\mathrm{pH}$ values (Fig. 2b). During the separation, analytes are trapped in the droplets with $\mathrm{pH}$ corresponding to their pI values, which is a favorable feature for preparative separations.

iii) The $\mathrm{pH}$ gradient for the IEF process is normally created in the electric field by the mixture of ampholytes. In an alternative approach, water electrolysis has been utilized for the formation of the
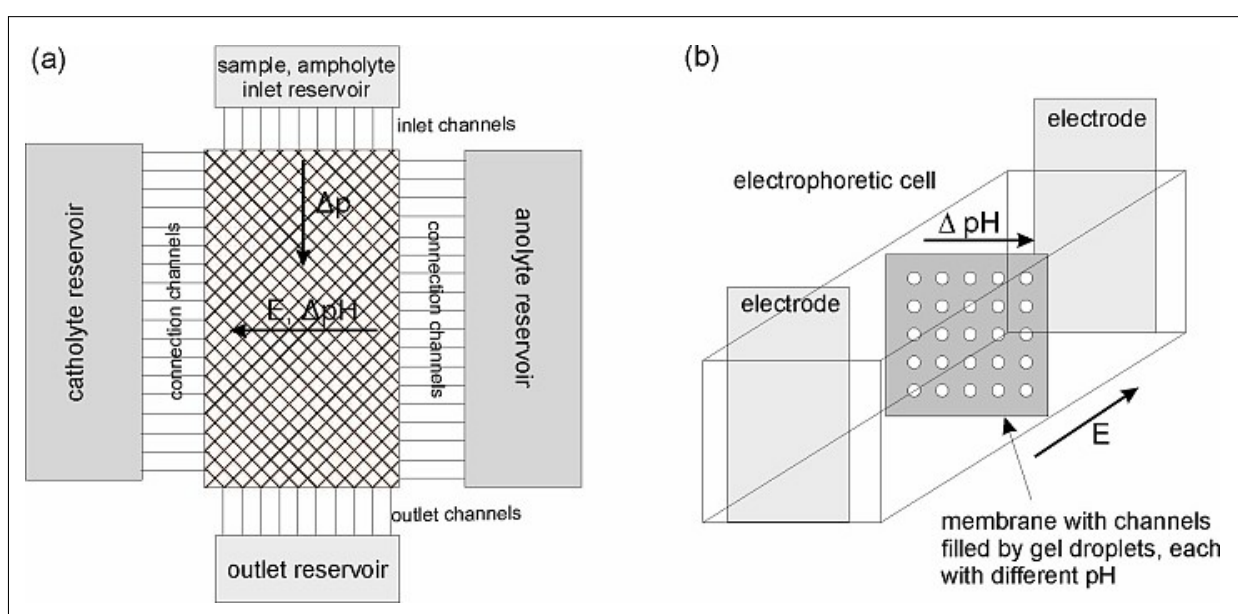

Fig. 2. Non-standard approaches to MC-IEF. Schematic drawing of (a) free flow MC-IEF; (b) parallel $\mathrm{MC}-\mathrm{IEF}$; the arrows indicate the direction of pressure gradient $(\Delta \mathrm{p})$, electric field $(\mathrm{E})$, and $\mathrm{pH}$ gradient $(\Delta \mathrm{pH})$.
$\mathrm{pH}$ gradient in a microfluidic device developed by Macounova et al. ${ }^{[38,39]}$ The separation channel of this device has the two opposite walls formed by gold or palladium electrodes. Upon application of an electric field to the electrodes, water electrolysis occurs and an electrically generated $\mathrm{pH}$ gradient is formed across the channel. The proteins are admitted to the channel in pressure-driven flow and are separated by means of transverse IEF (Fig. 3a). Another nonstandard approach represents a thermally generated $\mathrm{pH}$ gradient, which is formed in the electric field along a tapered separation channel filled with a buffer ${ }^{[27]}$ (Fig. 3b). Nonuniform Joule heating is generated along the tapered channel, which causes the formation of a $p H$ gradient by the buffer as its dissociation constant depends on the temperature.

\section{Conclusions and Outlook}

The feasibility of performing IEF on microchip has been demonstrated and MCIEF has become a new emerging analytical technology with a rapidly growing number of applications. Compared to the miniaturization of other electrophoretic techniques, the adaptation of IEF to microchip has the advantage of not being hindered by compromised resolution due to shorter separation paths. The resolution independence of the separation length is a unique feature of IEF and has been verified both experimentally and theoretically. Moreover, due to the specific experimental set-up of MC-IEF, the problematic cross injection, commonly employed for electrophoresis miniaturized to the microchip, could be avoided. The planar arrangement of the microchip also facilitates the implementation of whole-column imaged detection, which was identified as the most suitable detection mode for IEF separation. ${ }^{[40]}$ The successful coupling of MC-IEF to another separation process for 2D separation or to MS clearly demonstrates the potential of MC-IEF technology for system integration. Last but not least, MC-IEF accomplished in cheap plastic microchips shows great promise for the development of disposable chips for routine analyses.

Even though MC-IEF is still at the stage of testing the robustness of the technique for the separation of real protein samples, there is no doubt of its significant impact on

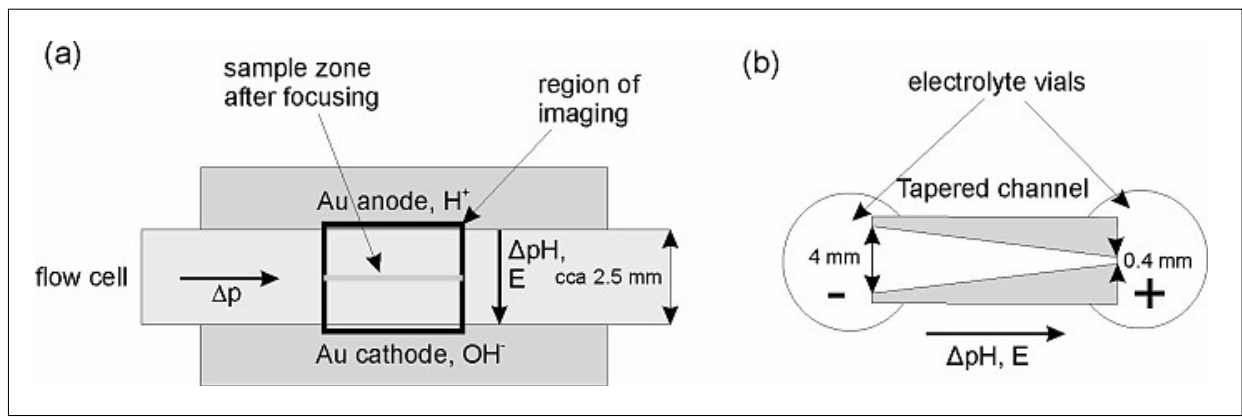

Fig. 3. Alternative ways of $\mathrm{pH}$ gradient formation. Schematic drawing of (a) electrically generated $\mathrm{pH}$ gradient; (b) thermally generated $\mathrm{pH}$ gradient; the arrows indicate the direction of pressure gradient $(\Delta \mathrm{p})$, electric field $(\mathrm{E})$, and $\mathrm{pH}$ gradient $(\Delta \mathrm{pH})$. 
protein analytics, in particular in the area of proteomics. Due to its high resolving power and spontaneous concentration of proteins, MC-IEF will presumably play an indispensable role in the integrated chip systems for protein analysis. The commercialization of easily operable microfabricated devices employing MC-IEF can be expected in the near future, leading to a revolution in many laboratories for routine analyses, especially in the pharmaceutics, medicine or life sciences.

\section{Acknowledgements:}

Partial funding for this project provided by Swiss National Science Foundation, grant number 200020-112190, is gratefully acknowledged.

Received: February 25, 2008

[1] R. Westermeier, 'Electrophoresis in practice', Wiley, Weinheim, 2001, p. 45.

[2] F. Kilar, Electrophoresis 2003, 24, 3908.

[3] Q. L. Mao, J. Pawliszyn, Analyst 1999, $124,637$.

[4] V. Dolnik, S. R. Liu, J. Sep. Sci. 2005, 28, 1994.

[5] J. Q. Wu, S. C. Li, A. Watson, J. Chromatogr. A 1998, 817, 163.

[6] B. Yao, H. H. Yang, Q. L. Liang, G. Luo, L. D. Wang, K. N. Ren, Y. D. Gao, Y. M. Wang, Y. Qiu, Anal. Chem. 2006, 78, 5845 .

[7] O. Hofmann, D. P. Che, K. A. Cruickshank, U. R. Muller, Anal. Chem. 1999, 71, 678.

[8] W. Tan, Z. H. Fan, C. X. Qiu, A. J. Ricco, I. Gibbons, Electrophoresis 2002, 23, 3638 .

[9] J. Han, A. K. Singh, J. Chromatogr. A 2004, 1049, 205.

[10] C. Das, Z. H. Fan, Electrophoresis 2006, 27, 3619.
[11] O. Vesterbe, H. Svensson, Acta Chem Scand. 1966, 20, 820.

[12] A. V. Stoyanov, C. Das, C. K. Fredrickson, Z. H. Fan, Electrophoresis 2005, 26, 473.

[13] H. C. Cui, K. Horiuchi, P. Dutta, C. F. Ivory, Anal. Chem. 2005, 77, 1303.

[14] M. Vlckova, F. Kalman, M. A. Schwarz, J. Chromatogr. A 2008; 1181, 145-152.

[15] F. Raisi, P. Belgrader, D. A. Borkholder, A. E. Herr, G. J. Kintz, F. Pourhamadi, M. T. Taylor, M. A. Northrup, Electrophoresis 2001, 22, 2291.

[16] J. C. Sanders, Z. L. Huang, J. P. Landers, Lab Chip 2001, 1, 167.

[17] C. Das, Z. Xia, A. Stoyanov, Z. H. Fan, Instrum. Sci.Technol. 2005, 33, 379.

[18] X. Y. Huang, J. C. Ren, Electrophoresis 2005, 26, 3595.

[19] C. Guillo, J. M. Karlinsey, J. P. Landers, Lab Chip 2007, 7, 112.

[20] J. Wen, Y. H. Lin, F. Xiang, D. W. Matson, H. R. Udseth, R. D. Smith, Electrophoresis 2000, $21,191$.

[21] M. L. S. Mok, L. Hua, J. B. C. Phua, M. K. T. Wee, N. S. K. Sze, Analyst 2004, 129, 109.

[22] M. Fujita, W. Hattori, T. Sano, M. Baba, H. Someya, K. Miyazaki, K. Kamijo, K. Takahashi, H. Kawaura, J. Chromatogr. A 2006, 1111, 200.

[23] Y. Li, D. L. DeVoe, C. S. Lee, Electrophoresis 2003, 24, 193.

[24] A. Griebel, S. Rund, F. Schonfeld, W. Dorner, R. Konrad, S. Hardt, Lab Chip 2004, 4,18 .

[25] Y. Li, J. S. Buch, F. Rosenberger, D. L. DeVoe, C. S. Lee, Anal. Chem. 2004, 76 , 742.

[26] C. Li, Y. N. Yang, H. G. Craighead, K. H. Lee, Electrophoresis 2005, 26, 1800.

[27] T. M. Huang, J. Pawliszyn, Electrophoresis 2002, 23, 3504.

[28] S. W. Tsai, M. Loughran, A. Hiratsuka, K. Yano, I. Karube, Analyst 2003, 128, 237.
[29] A. E. Herr, J. I. Molho, K. A. Drouvalakis, J. C. Mikkelsen, P. J. Utz, J. G. Santiago, T. W. Kenny, Anal. Chem. 2003, 75, 1180.

[30] Y. C. Wang, M. N. Choi, J. Y. Han, Anal. Chem. 2004, 76, 4426.

[31] H. C. Cui, K. Horiuchi, P. Dutta, C. F. Ivory, Anal. Chem. 2005, 77, 7878.

[32] Y. Xu, C. X. Zhang, D. Janasek, A. Manz, Lab Chip 2003, 3, 224.

[33] D. Kohlheyer, G. A. J. Besselink, S. Schlautmann, R. B. M. Schasfoort, Lab Chip 2006, 6, 374.

[34] D. Kohlheyer, J. C. T. Eljkel, S. Schlautmann, A. van den Berg, R. B. M. Schasfoort, Anal. Chem. 2007, 79, 8190.

[35] G. V. Zilberstein, E. M. Baskin, S. Bukshpan, Electrophoresis 2003, 24, 3735.

[36] G. V. Zilberstein, E. M. Baskin, S. Bukshpan, L. E. Korol, Electrophoresis 2004, 25, 3643.

[37] G. Zilberstein, L. Korol, S. Bukshpan, E. Baskin, Proteomics 2004, 4, 2533.

[38] K. Macounova, C. R. Cabrera, P. Yager, Anal. Chem. 2001, 73, 1627.

[39] K. Macounova, C. R. Cabrera, M. R. Holl, P. Yager, Anal. Chem. 2000, 72, 3745.

[40] X. Z. Wu, J. Q. Wu, J. Pawliszyn, $L C-G C$ 2001, 19, 527. 